\title{
Clinical Study \\ Robotically Assisted Hysterectomy versus Vaginal Hysterectomy for Benign Disease: A Prospective Study
}

\author{
M. Carbonnel, H. Abbou, H. T. N'Guyen, S. Roy, G. Hamdi, A. Jnifen, and J. M. Ayoubi \\ Service de Gynécologie Obstetrique, Hôpital Foch, Faculté de Médecine Paris Ouest, 92150 Suresnes, France \\ Correspondence should be addressed to M. Carbonnel; carbonnelmarie@gmail.com
}

Received 7 April 2013; Accepted 3 June 2013

Academic Editor: Peng Hui Wang

Copyright ( 2013 M. Carbonnel et al. This is an open access article distributed under the Creative Commons Attribution License, which permits unrestricted use, distribution, and reproduction in any medium, provided the original work is properly cited.

\begin{abstract}
Objectives. A prospective study was carried out to compare vaginal hysterectomy ( $\mathrm{VH}$ ) and robotically assisted hysterectomy (RH) for benign gynecological disease. Materials and Methods. All patients who underwent hysterectomy from March 2010 to March 2012 for a benign disease were included. Patients' demographics per and post surgery results were collected from medical files. A questionnaire was also conducted 2 months after surgery. Results. Sixty patients were included in the RH group and thirty four in the VH one. Operative time was significantly longer in the RH group $(132.1 \pm 5.7$ versus $75.3 \pm 6.7 \mathrm{~min} ; P<0.0001)$. Blood loss and length of hospital stay were significantly reduced: $47 \pm 7$ versus $125 \pm 20 \mathrm{ml} ; P<0.01$, and $2.4 \pm 0.1$ versus $3.3 \pm 0.2$ days; $P<0.0001$, respectively. Less pain was reported at D1 and D2 by RH patients, and levels of analgesia were lower compared to those observed in the VH group. No differences were found regarding the rate of conversion to laparotomy, intra- or postoperative complications. Conclusion. Robotically assisted hysterectomy appears to reduce blood loss, postoperative pain, and length of hospital stay, but it is associated with longer operative time and higher cost. Specific indications for RH remain to be defined.
\end{abstract}

\section{Introduction}

Many techniques of hysterectomy are being used in the surgical treatment of benign gynecological disease. Laparotomy is still the commonest and the easiest one, yet it is the most invasive [1]. Abdominal laparoscopic hysterectomy and vaginal hysterectomy $(\mathrm{VH})$ should be preferred because they are minimally invasive. $\mathrm{VH}$ does not leave scars and it can be used in obese patients. However, it may be difficult in cases of enlarged uterus, nulliparous women, and in patients with pelvic adhesions; adnexectomy may also fail in case of upper abdominal adnexal masses [2]. Total laparoscopic hysterectomy reduces blood loss and postoperative pain; it is easer to make adnexectomy and adhesiolysis and is feasible in nulliparous women [3]. Laparoscopically assisted VH allows performing adnexectomy and adhesiolysis in hysterectomic procedures ended by vaginal approach which simplifies the laparoscopic time; however, such procedure is associated with greater postoperative discomfort compared to vaginal procedure $[4,5]$. Robotically assisted surgery appears "pleasant" in this indication, overcoming the laparoscopic hysterectomy limits. The rotation of the instruments, the $3 \mathrm{D}$ visual approach, tremor reduction, operating comfort, and the intuitive pattern of the da Vinci robot significantly simplify surgical gestures. The da Vinci robot has been authorized for hysterectomy procedures in 2005 in the United States; hysterectomy is currently one of the most common surgical interventions worldwide [6]. To date, very few studies have compared the outcomes of robotically assisted hysterectomy (RH) and $\mathrm{VH}$ in benign gynecological disease. This paper presents our prospective comparison of these two approaches.

\section{Methods}

We carried out in our department of gynecology and obstetrics (Foch Hospital, Suresnes, France) a 2-year prospective study, from March 2010 to March 2012. All hysterectomies done for benign gynecological disease were included: $60 \mathrm{RH}$ and $34 \mathrm{VH}$. Patients' demographics and medical characteristics were collected from the medical files: age, BMI, surgical indication, surgical history, menopausal status, and hormone replacement therapy were studied, as well as operative time, 
TABLE 1: Demographics and characteristics of the population expressed as mean \pm SD and number (\%).

\begin{tabular}{lccc}
\hline & $\mathrm{RH}(n=60)$ & $\mathrm{VH}(n=34)$ & $P$ \\
\hline Age (years) & $43.7 \pm 1.7$ & $51.2 \pm 1.6$ & 0.06 \\
$\mathrm{BMI}\left(\mathrm{kg} / \mathrm{m}^{2}\right)$ & $24.8 \pm 0.5$ & $26.5 \pm 1.3$ & $\mathrm{NS}$ \\
Gestity & $1.7 \pm 0.3$ & $2.7 \pm 0.3$ & 0.02 \\
Parity & $1.3 \pm 0.2$ & $2.3 \pm 0.2$ & 0.01 \\
Menopause & $14(23 \%)$ & $9(26 \%)$ & $\mathrm{NS}$ \\
HRT & $7 / 14(50 \%)$ & $2 / 9(22 \%)$ & $\mathrm{NS}$ \\
History of laparotomy & $17(28 \%)$ & $7(20 \%)$ & $\mathrm{NS}$ \\
Nonconservative hysterectomy & $43(72 \%)$ & $19(29 \%)$ & 0.02 \\
Interadnexal hysterectomy & $17(28 \%)$ & $24(71 \%)$ & 0.01 \\
\hline
\end{tabular}

SD: standard deviation, NS: nonsignificant, HRT: hormone replacement therapy.

docking time, anesthesia, uterine weight, blood loss, transfusions, conversion to laparotomy, intra- and postoperative complications, and pre- and postoperative hemoglobin.

Two operators performed the RH and seven the VH. $\mathrm{VH}$ procedures were conventionally carried out with vicryl ligatures; $\mathrm{RH}$ procedures were performed using a uterine manipulator, and vaginal suturing was done using vicryl 1 continuous or interrupted sutures. A questionnaire was completed by all patients postoperatively, aimed to evaluate their pain at D0, D1, D2, and D3 using a visual analog rating 010 scale. The levels of analgesia, total morphine consumption, transit recovery delay, and the length of hospital stay (number of days) were also reported. Two months after surgery a questionnaire was completed by the patients regarding the duration of their work cessation, time to return to normal life, postoperative complications, pain, sexual life (unchanged, improved, or deteriorated compared with presurgery), and overall satisfaction regarding the intervention (dissatisfied, fairly satisfied, satisfied, and very satisfied).

Quantitative variables were compared using nonparametric tests, and sample comparisons were performed by chisquare tests. The level of significance was $P<0.05$.

\section{Results}

Patients' characteristics are displayed in Table 1 . In the $\mathrm{VH}$ group, indications were metrorrhagia associated with fibroma or endometrial hypertrophy in 26 cases, atypical hyperplasia in 4 cases, and high-grade dysplasia in 4 cases. Among $\mathrm{RH}$ patients there was a Benjamin syndrome in 20 cases, metrorrhagia induced by fibroma or endometrial hypertrophy in 27 cases, pain associated with adenomyosis in 3 cases, and atypical hyperplasia in 10 cases. To note that, patients were randomly classified into either group, except for those having Benjamin syndrome that were included in the RH arm.

Intraoperative data are displayed in Table 2. In the $\mathrm{VH}$ group, 2 patients underwent transfusion of 1 and 3 packed red blood cells (the 2 complications reported in this group) and 1 had laparoconversion induced by hemorrhage. In the $\mathrm{RH}$ group, the 2 reported complications were 1 bladder injury
TABLE 2: Interoperative results expressed as mean \pm SD and number (\%).

\begin{tabular}{lccc}
\hline & $\mathrm{RH}(n=60)$ & $\mathrm{VH}(n=34)$ & $P$ \\
\hline pAnesthesia time (min) & $195.8 \pm 6.4$ & $115.3 \pm 7$ & $<0.0001$ \\
Operative time (min) & $132.1 \pm 5.7$ & $75.3 \pm 6.7$ & $<0.0001$ \\
Trocar placement time (min) & $9.5 \pm 0.5$ & & \\
Docking time (min) & $7 \pm 0.6$ & & \\
Console time (min) & $94 \pm 6$ & & \\
Uterine weight (g) & $136 \pm 14$ & $226.7 \pm 31.6$ & 0.004 \\
Blood loss (mL) & $47 \pm 7$ & $125 \pm 20$ & $<0.01$ \\
Transfusions & 0 & $2(6 \%)$ & NS \\
Laparoconversion & 0 & 1 & NS \\
Complications & $2(3 \%)$ & $2(8 \%)$ & NS \\
\hline
\end{tabular}

SD: standard deviation, min: minutes, $\mathrm{mL}$ : milliliters, g: grams, NS: nonsignificant.

that occurred while detaching the vesicouterine cul-de-sac (patient with a history of 3 cesarean sections) and 1 injury of the small intestinal serosa that occurred during open laparoscopy, sutured by one vicryl 2.0. Five adhesiolyses were carried out in this group.

Table 3 presents the postoperative results until hospital discharge. At D3, 7 patients had left hospital in the VH group (20\%) and 23 in the RH group (40\%). No postoperative complications were reported in the $\mathrm{VH}$ group while 1 occurred in one $\mathrm{RH}$ patient: an abscess of Douglas pouch occurring 10 days after surgery and necessitating antibiotherapy along with a 5-day hospital stay without surgical reintervention.

The results obtained by the questionnaire completed 2 months after surgery are displayed in Table 4 . In the $\mathrm{VH}$ group 28 questionnaires (82\%) have been completed and 41 (70\%) in the RH one. No difference was observed between the two groups regarding sexual life. In the VH group, among the 16 patients reporting a sexual activity before and after surgery, 8 evaluated it as unchanged, 4 worsened, and 4 improved. In the $\mathrm{RH}$ group, among the 20 patients reporting a sexual activity before and after surgery, 16 evaluated it as unchanged, 1 worsened, and 3 improved.

\section{Discussion}

Robotically assisted surgery offers advantages over laparoscopy in hysterectomy procedures for benign disease. The princeps series of Payne and Dauterive [7] showed beneficial results regarding uterine weight, operative time in the 25 last procedures (series of 100 cases), blood loss, laparoconversions, and hospital stay duration. This author confirmed such results in a meta-analysis [8]. The rate of vaginal cuff dehiscence has been probably overestimated in the first series [9]; it appears to be $1.5 \%$ like that observed with laparoscopy [10]. We had no cases of dehiscence in our series: only one case of pelvic abscess that resolved after antibiotherapy.

A comparative study of $\mathrm{RH}$ and laparoscopic-assisted $\mathrm{VH}$ showed that the robotic procedure reduces the operative time and duration of hospital stay with less blood loss [11]. Very few studies have compared RH and $\mathrm{VH}$ [12-15]. Matthews et al. 
TABLE 3: Postoperative results expressed as mean \pm SD and number (\%).

\begin{tabular}{lccc}
\hline & $\mathrm{RH}(n=60)$ & $\mathrm{VH}(n=34)$ & $P$ \\
\hline Pain on the VAS, D0 & $4.7 \pm 0.4$ & $5.1 \pm 0.4$ & $\mathrm{NS}$ \\
Analgesic level, D0 & $2.5 \pm 0.1$ & $2.3 \pm 0.1$ & $\mathrm{NS}$ \\
Pain on the VAS, D1 & $3.1 \pm 0.3$ & $4 \pm 0.3$ & 0.03 \\
Analgesic level, D1 & $1.8 \pm 0.4$ & $1.8 \pm 0.1$ & $\mathrm{NS}$ \\
Pain on the VAS, D2 & $2.2 \pm 0.3$ & $3.2 \pm 0.3$ & 0.04 \\
Analgesic level, D2 & $1.3 \pm 0.1$ & $1.7 \pm 0.1$ & 0.04 \\
Pain on the VAS, D3 & $1.8 \pm 0.3$ & $2.3 \pm 0.2$ & $\mathrm{NS}$ \\
$\begin{array}{l}\text { Analgesic level, D3 } \\
\text { Time to transit return (days) }\end{array}$ & $1.2 \pm 0.2$ & $1.4 \pm 0.1$ & $\mathrm{NS}$ \\
$\begin{array}{l}\text { Total morphine consumption } \\
\text { (mg) }\end{array}$ & $7.9 \pm 1.4$ & $1.5 \pm 0.1$ & $\mathrm{NS}$ \\
$\begin{array}{l}\text { Difference in hemoglobin } \\
\text { level (g/dL) }\end{array}$ & $1.2 \pm 0.1$ & $0.9 \pm 0.1$ & $\mathrm{NS}$ \\
$\begin{array}{l}\text { Duration of hospital stay } \\
\text { (days) }\end{array}$ & $2.4 \pm 0.1$ & $3.3 \pm 0.2$ & $<0.0001$ \\
\hline
\end{tabular}

VAS: visual analog scale, SD: standard deviation, dl: deciliters, g: grams, NS: nonsignificant.

TABLE 4: Results of the questionnaire completed 2 months after surgery expressed as mean \pm SD and number (\%).

\begin{tabular}{lccc}
\hline & $\mathrm{RH}(n=60)$ & $\mathrm{VH}(n=34)$ & $P$ \\
\hline Duration of work cessation (days) & $30.9 \pm 2.5$ & $35.9 \pm 3.5$ & $\mathrm{NS}$ \\
Return to normal life (days) & $20.7 \pm 2.9$ & $17.9 \pm 3.3$ & $\mathrm{NS}$ \\
Dyspareunia & $1 / 20(5 \%)$ & $2 / 17(12 \%)$ & $\mathrm{NS}$ \\
Pelvic pain & $12(29 \%)$ & $9(28 \%)$ & $\mathrm{NS}$ \\
Analgesic level & $0.6 \pm 0.1$ & $0.5 \pm 0.1$ & $\mathrm{NS}$ \\
Very satisfied & $31(75 \%)$ & $22(70 \%)$ & $\mathrm{NS}$ \\
Satisfied & $6(15 \%)$ & $6(18 \%)$ & $\mathrm{NS}$ \\
Fairly satisfied & $2(5 \%)$ & $2(6 \%)$ & NS \\
Dissatisfied & $2(5 \%)$ & $2(6 \%)$ & NS \\
\hline
\end{tabular}

NS: nonsignificant.

carried out a retrospective analysis of the various surgical approaches used in their department during the first year after robotic equipment was introduced in this unit [12]. They observed beneficial results associated with the robot regarding blood loss, transfusion rate, and infection rate. In another retrospective series, Landeen et al. [15] compared all surgical approaches for hysterectomy; they underline less blood loss with the robot and reduced hospital stay, while $\mathrm{VH}$ was associated with a shortened operative time and reduced cost of the procedure. The two other comparisons were reported in congress abstracts $[13,14]$.

We found no randomized study or prospective study on this comparison. Our results are in accordance with those reported in the literature regarding blood loss and duration of hospital stay. We observed also a lengthened operative time with the robotic procedure. Our study reports no significant difference between the two procedures regarding intra- and postoperative complications; in fact, hysterectomy in benign disease is usually associated with a low incidence of complications, and no difference could be evidenced with such small sample.

We also focused on postoperative pain, a fact poorly present in the literature, although some authors have underlined the beneficial outcome of laparoscopy over $\mathrm{VH}$ regarding postoperative pain [4]. Our study reports less postoperative pain associated with the robotic approach at D1 and D2 on the rating scale and lower analgesic level at D2. Such results were not seen at D3, probably biased by the discharge of a great number of $\mathrm{RH}$ patients at $\mathrm{D} 2$. We observed no difference in terms of morphine consumption. Morphinelike agents are primarily used in the recovery room and may have been overused at first interventions carried out in the department, making our results possibly biased. The questionnaire completed 2 months after surgery shows no significant differences between groups and reveals a significant number of lost-to-follow-up patients. The main bias of our series is the lack of randomization of all patients. In fact, populations were different with younger patients, lower parity data, and more frequent nonconservative hysterectomies in the RH group. This bias was due to surgical indications. Benjamin syndromes were young and had smaller uterus. But they were nullipara, and it was very important for them to undergo ovariectomy. So hysterectomy was robotically assisted for this indication in all cases (1/3 of indications of $\mathrm{RH}$ group) in order to avoid laparotomy. Therefore we have to continue evaluation in the future with information collected prospectively and probably with randomized methodology We have not studied the related costs, although this represents a major disadvantage of the robotic surgery. The costs related to robotic surgery are higher than those related to the laparoscopic and vaginal approaches [16] but lower than laparotomy-related operative cost.

The advantages presented by the robotic surgery over the vaginal approach in hysterectomy are counterbalanced by its higher operative cost and lengthened operative time. To date, it does not seem reasonable to systematically use robotics in all hysterectomies, but the robotic procedure presents significant interest in that it allows preventing laparotomy and laparoscopic-assisted VH. Such technique could be considered in complex diseases (enlarged uterine volumes, obese patients, etc.) [17] until the reduction of its cost which should help its diffusion.

\section{References}

[1] T. E. Nieboer, N. Johnson, A. Lethaby et al., "Surgical approach to hysterectomy for benign gynaecological disease," Cochrane Database of Systematic Reviews, no. 3, Article ID CD003677, 2009.

[2] E. David-Montefiore, R. Rouzier, C. Chapron, E. Daraï, and Collegiale d'Obstétrique et Gynécologie de Paris-Ile de France, "Surgical routes and complications of hysterectomy for benign disorders: a prospective observational study in French university hospitals," Human Reproduction, vol. 22, no. 1, pp. 260-265, 2007.

[3] R. Gendy, C. A. Walsh, S. R. Walsh, and E. Karantanis, "Vaginal hysterectomy versus total laparoscopic hysterectomy for benign disease: a metaanalysis of randomized controlled 
trials," The American Journal of Obstetrics and Gynecology, vol. 204, no. 5, pp. 388.e1-388.e8, 2011.

[4] F. Ghezzi, S. Uccella, A. Cromi et al., "Postoperative pain after laparoscopic and vaginal hysterectomy for benign gynecologic disease: a randomized trial," The American Journal of Obstetrics and Gynecology, vol. 203, no. 2, pp. 118.el-118.e8, 2010.

[5] J. Drahonovsky, L. Haakova, M. Otcenasek, L. Krofta, E. Kucera, and J. Feyereisl, "A prospective randomized comparison of vaginal hysterectomy, laparoscopically assisted vaginal hysterectomy, and total laparoscopic hysterectomy in women with benign uterine disease," European Journal of Obstetrics Gynecology and Reproductive Biology, vol. 148, no. 2, pp. 172$176,2010$.

[6] L. Weinberg, S. Rao, and P. F. Escobar, "Robotic surgery in gynecology: an updated systematic review," Obstetrics and Gynecology International, vol. 2011, Article ID 852061, 29 pages, 2011.

[7] T. N. Payne and F. R. Dauterive, "A comparison of total laparoscopic hysterectomy to robotically assisted hysterectomy: surgical outcomes in a community practice," Journal of Minimally Invasive Gynecology, vol. 15, no. 3, pp. 286-291, 2008.

[8] T. N. Payne and M. C. Pitter, "Robotic-assisted surgery for the community gynecologist: can it be adopted?" Clinical Obstetrics and Gynecology, vol. 54, no. 3, pp. 391-411, 2011.

[9] R. M. Kho, M. N. Akl, J. L. Cornella, P. M. Magtibay, M. E. Wechter, and J. F. Magrina, "Incidence and characteristics of patients with vaginal cuff dehiscence after robotic procedures," Obstetrics and Gynecology, vol. 114, no. 2, pp. 231-235, 2009.

[10] G. Gaia, R. W. Holloway, L. Santoro, S. Ahmad, E. Di Silverio, and A. Spinillo, "Robotic-assisted hysterectomy for endometrial cancer compared with traditional laparoscopic and laparotomy approaches: a systematic review," Obstetrics and Gynecology, vol. 116, no. 6, pp. 1422-1431, 2010.

[11] B. N. Giep, H. N. Giep, and H. B. Hubert, "Comparison of minimally invasive surgical approaches for hysterectomy at a community hospital: robotic-assisted laparoscopic hysterectomy, laparoscopic-assisted vaginal hysterectomy and laparoscopic supracervical hysterectomy," Journal of Robotic Surgery, vol. 4, no. 3, pp. 167-175, 2010.

[12] C. A. Matthews, N. Reid, V. Ramakrishnan, K. Hull, and S. Cohen, "Evaluation of the introduction of robotic technology on route of hysterectomy and complications in the first year of use," The American Journal of Obstetrics and Gynecology, vol. 203, no. 5, pp. 499.e2-499.e5, 2010.

[13] E. Jacome, "Robotic assisted hysterectomy versus vaginal hysterectomy for benign gynecologic conditions. A comparative analysis of surgical outcomes," Journal of Minimally Invasive Gynecology, vol. 17, no. 6, p. S26, 2010.

[14] K. N. Wright, G. M. Jonsdottir, S. Jorgensen, and J. J. Einarsso, "A comparison of abdominal, laparoscopic, vaginal, and robotic hysterectomies: surgical outcomes and operative cost in a single institution," Journal of Minimally Invasive Gynecology, vol. 17, Supplement 1, no. 6, p. S23, 2010.

[15] L. B. Landeen, M. C. Bell, H. B. Hubert, L. Y. Bennis, S. S. Knutsen-Larson, and U. Seshadri-Kreaden, "Clinical and cost comparisons for hysterectomy via abdominal, standard laparoscopic, vaginal and robot-assisted approaches," South Dakota Medicine, vol. 64, no. 6, pp. 197-203, 2011.

[16] R. P. Pasic, J. A. Rizzo, H. Fang, S. Ross, M. Moore, and C. Gunnarsson, "Comparing robot-assisted with conventional laparoscopic hysterectomy: impact on cost and clinical outcomes," Journal of Minimally Invasive Gynecology, vol. 17, no. 6, pp. 730-738, 2010.

[17] J. F. Boggess, P. A. Gehrig, L. Cantrell et al., "Perioperative outcomes of robotically assisted hysterectomy for benign cases with complex pathology," Obstetrics and Gynecology, vol. 114, no. 3, pp. 585-593, 2009. 


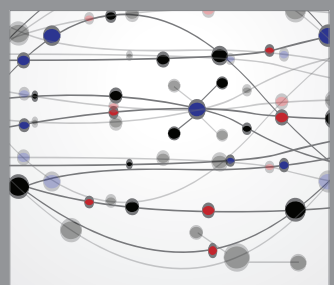

The Scientific World Journal
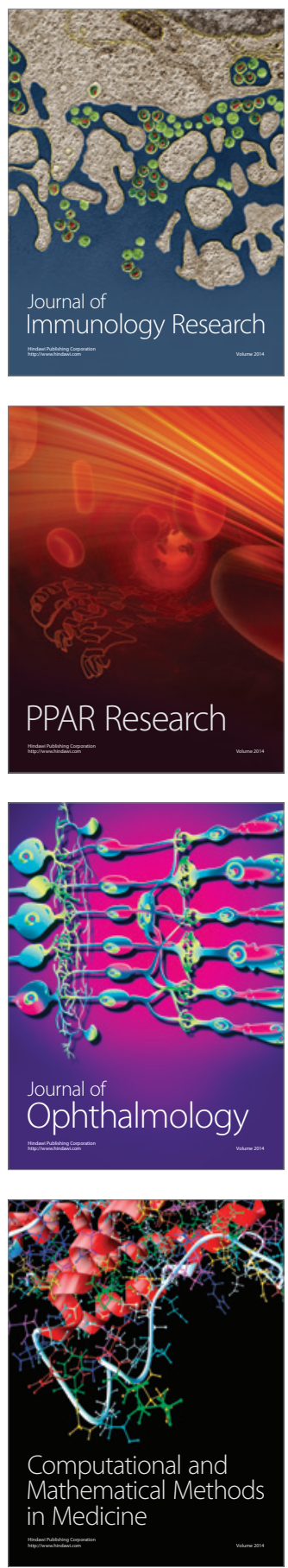

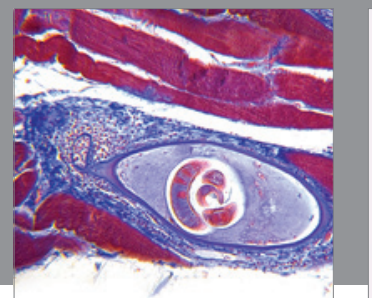

Gastroenterology

Research and Practice
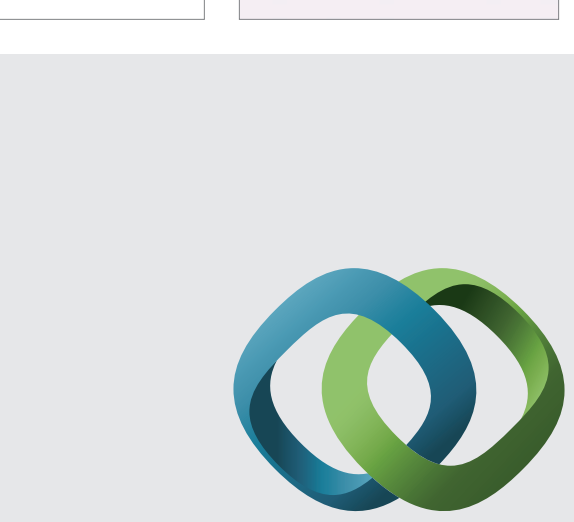

\section{Hindawi}

Submit your manuscripts at

http://www.hindawi.com
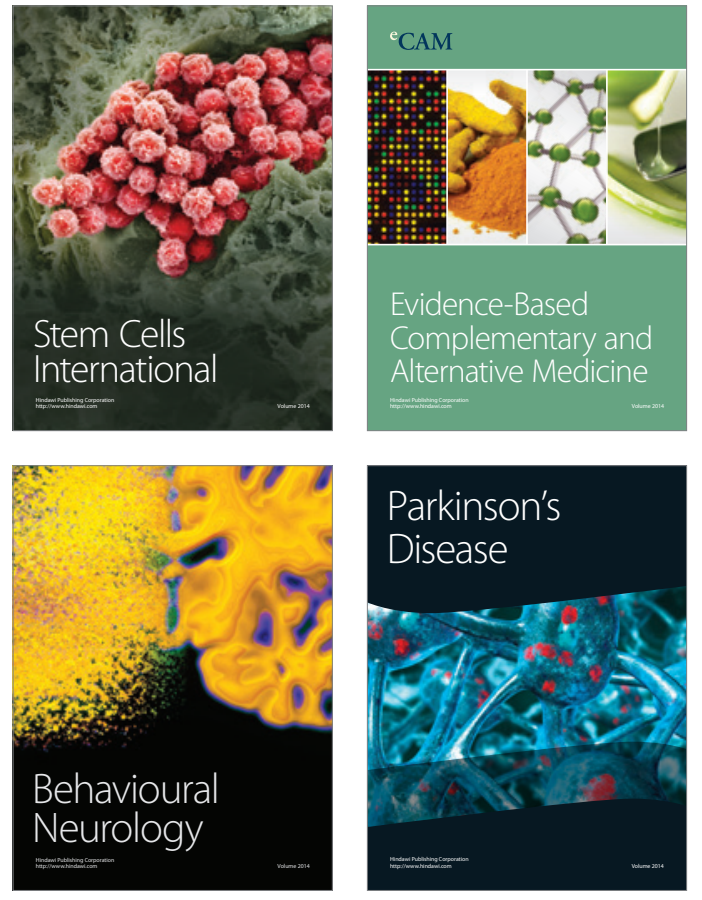
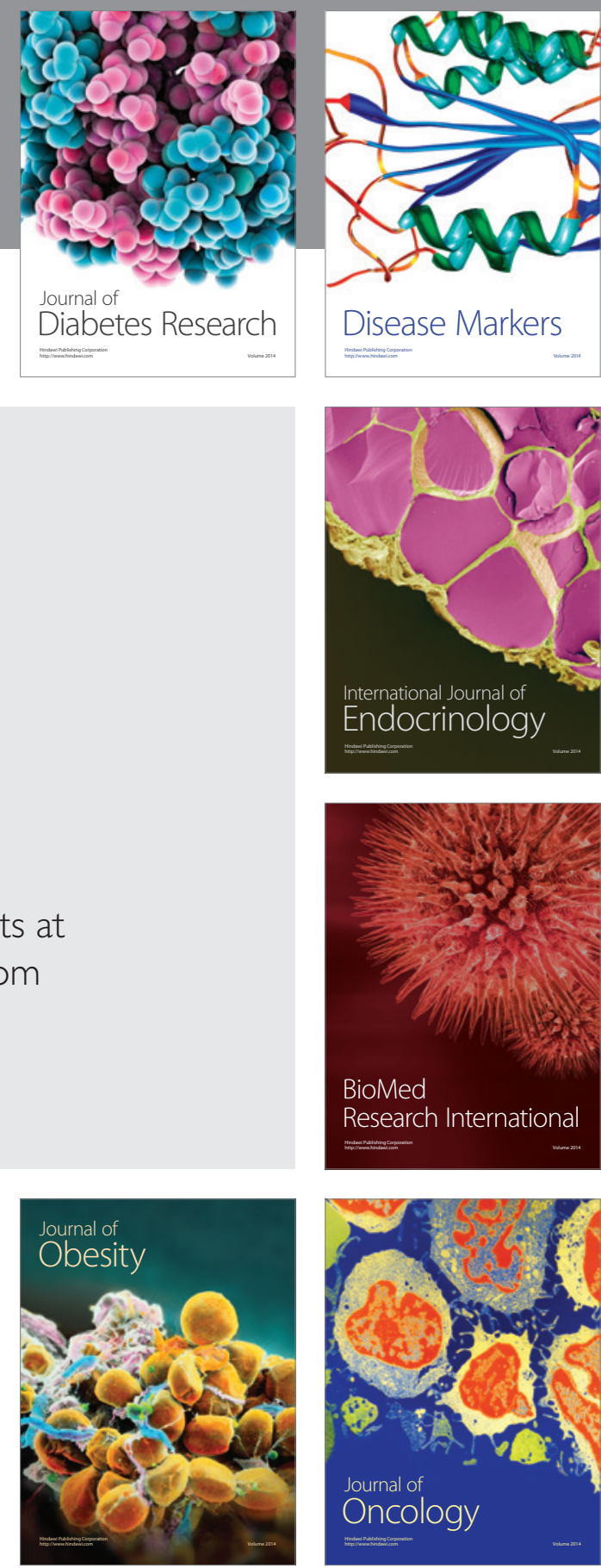

Disease Markers
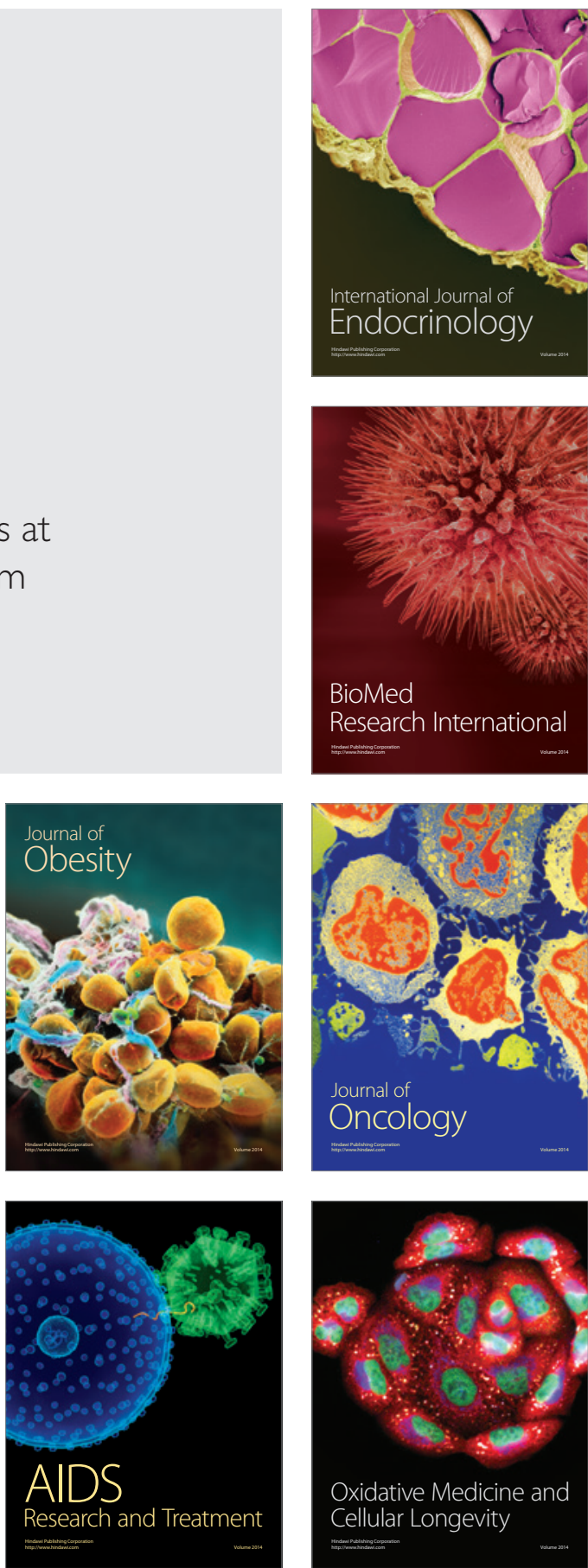